\title{
The impact of EGFR exon 19 deletion subtypes on clinical outcomes in non-small cell lung cancer
}

\author{
Chao Zhao" ${ }^{1 \#}$, Tao Jiang ${ }^{2 \#}$, Jiayu $\mathrm{Li}^{2 \#}$, Yan Wang ${ }^{2 \#}$, Chunxia $\mathrm{Su}^{2}$, Xiaoxia Chen ${ }^{2}$, Shengxiang Ren ${ }^{2}, \mathrm{Xuefei} \mathrm{Li}^{1}$, \\ Caicun Zhou ${ }^{2}$ \\ ${ }^{1}$ Department of Lung Cancer and Immunology, ${ }^{2}$ Department of Medical Oncology, Shanghai Pulmonary Hospital, Tongji University, Shanghai, \\ China \\ Contributions: (I) Conception and design: C Zhao, T Jiang; (II) Administrative support: X Li, C Zhou; (III) Provision of study materials or patients: \\ C Su, X Chen, S Ren; (IV) Collection and assembly of data: J Li, Y Wang; (V) Data analysis and interpretation: C Zhao, T Jiang; (VI) Manuscript \\ writing: All authors; (VII) Final approval of manuscript: All authors. \\ "These authors contributed equally to this work. \\ Correspondence to: Caicun Zhou. Department of Medical Oncology, Shanghai Pulmonary Hospital, Tongji University, No. 507, Zhengmin Road, \\ Shanghai 200433, China. Email: caicunzhoudr@163.com; Xuefei Li. Department of Lung Cancer and Immunology, Shanghai Pulmonary Hospital, \\ Tongji University, No. 507, Zhengmin Road, Shanghai 200433, China. Email: bug_lily2003@163.com.
}

Background: The study investigated the resistant pattern and clinical outcomes of epidermal growth factor receptor (EGFR) exon 19 deletion (19del) subtypes to tyrosine kinase inhibitors (TKIs) in non-small cell lung cancer (NSCLC).

Methods: Two hundred eight treatment naive NSCLC patients detected as EGFR 19del using amplification-refractory mutation system (ARMS) were included. DNA sequencing was used to detect the subtypes. Clinicopathological features as well as patients' outcomes treated with first-line EGFR-TKIs were analyzed.

Results: Thirteen EGFR 19del subtypes were confirmed in 181 samples (87.0\%). Among these, delE746_ A750 was the most frequent subtype (130/181, 71.8\%). delE746_A750 and deletions starting from E746 were frequently found in female $(\mathrm{P}=0.003$ and $\mathrm{P}=0.013$, respectively) and never smokers $(\mathrm{P}=0.002$ and $\mathrm{P}=0.014$, respectively) than non-delE746_A750 and deletions starting from L747 patients, respectively. T790M was more frequently occurred in delE746_A750 than non-delE746_A750 (P=0.001) and deletions starting from E746 than $\mathrm{L} 747$ patients $(\mathrm{P}=0.006)$ after first-line EGFR-TKIs resistance. Patients harboring deletions starting from L747 with insertions had significantly shorter progression-free survival (PFS) than deletions starting from $\mathrm{L} 747$ without insertion ( 8.3 vs. $15.0 \mathrm{~m}, \mathrm{P}=0.017$ ), or all other patients (8.3 vs. $12.6 \mathrm{~m}, \mathrm{P}=0.027)$. Different 19del subtypes with T790M mutation had similar PFS when treated with osimertinib ( $\mathrm{P}=0.102)$.

Conclusions: Patients with EGFR 19del subtypes had different clinicopathological features, and resistant pattern when treated with first-line TKIs. Patients harboring deletions starting from L747 with insertions had inferior outcomes than other subtypes.

Keywords: Epidermal growth factor receptor (EGFR); exon 19 deletion (19del); non-small cell lung cancer (NSCLC)

Submitted Aug 25, 2019. Accepted for publication Jun 10, 2020.

doi: $10.21037 /$ tlcr-19-359

View this article at: http://dx.doi.org/10.21037/tlcr-19-359 


\section{Introduction}

Targeted therapy for epidermal growth factor receptor (EGFR) mutation in non-small cell lung cancer (NSCLC) developed quickly (1-5). EGFR exon 19 deletion (19del) was about $44 \%$ in EGFR mutations, and the most frequent subtype was delE756_A750, followed by delL747_P753insS, delL747_A750insP or delL747_T751 (6,7). Studies reported that the $19 \mathrm{del}$ subtypes could have different survival outcomes to EGFR-tyrosine kinase inhibitors (EGFR-TKIs) (8-13). Chung et al. found that response rate (RR) was significantly different between non-LRE (codons L747 to E749) deletions and other deletions (8), while others $(10,12)$ did not find significant differences between the subtypes. Lee et al., Kaneda et al. and Sutiman et al. found that there were significant differences in progression-free survival (PFS) or overall survival (OS) between different EGFR 19del subtypes (9-11), however, it was not found in other studies $(8,12,13)$. Truini et al. also found that a deletion subtype starting from L747 with insertion (delL747_A750insP) had different sensitivities to different EGFR-TKIs (14). So, the association is still fuzziness and worth further study between 19del subtypes and patient clinical outcomes.

EGFR T790M mutation occurred in about $60 \%$ when were resistant to first generation EGFR-TKIs $(15,16)$. Studies reported that the incidence was higher in EGFR 19 del than L858R patients $(17,18)$. Besides, T790M might also distribute differently between EGFR 19del subtypes when patients are resistant to EGFR-TKIs (19). Osimertinib was efficient for T790M mutation patients. Whether it had different efficacy on the patients harboring T790M mutation in different EGFR 19del subtypes also needed to be studied. In the retrospective study, we explored the clinical characteristics and outcomes of patients harboring different EGFR 19del subtypes treated with firstline EGFR-TKIs, in order to better understand the impact of mutation subtypes to patients' outcomes. We present the following article in accordance with the STROBE reporting checklist (available at http://dx.doi.org/10.21037/tlcr19-359).

\section{Methods}

\section{Patients}

From April 2016 to August 2017, stage IIIB/IV Chinese NSCLC patients at diagnosis had EGFR/KRAS/BRAF/
ALK/ROS1 gene mutation detection. All patients provided written informed consent before molecular detection. Among these, 208 patients harboring only EGFR 19del conducted DNA sequencing to detect the subtypes. Patients using gefitinib, erlotinib, icotinib, or afatinib as first-line treatment was conducted for subsequent analysis. Osimertinib used for patients harboring EGFR T790M was analyzed in this study. Clinical outcomes were followed up until April 2019. This study was approved by the Ethics Committee of Shanghai Pulmonary Hospital (K19-066Y).

\section{Gene mutation detection}

Formalin-fixed paraffin-embedded (FFPE), fine/core needle aspiration, biopsy, or pleural effusion samples were used for the detection of alterations in the five genes. The detection using amplification-refractory mutation system (ARMS) was conducted as we had reported in previous studies $(20,21)$. EGFR mutation was detected by EGFR 29 Mutations Detection Kit (Amoy Diagnostics, Xiamen, China) as the protocol described, which covered $2519 \mathrm{del}$ subtypes (Table S1). After patients were resistant to EGFR-TKIs, peripheral blood, fine/core needle aspiration, biopsy, or pleural effusion samples were used to detect EGFR T790M mutation using EGFR 29 Mutations Detection Kit.

\section{Statistical analysis}

All statistical analyses were performed using the SPSS v.20 software (SPSS Inc., Chicago, IL, USA). Clinicopathological features were evaluated by Pearson Chi-square or Fisher's exact test. Kaplan-Meier method and cox-regression were used for survival analysis. The two-sided significance level was set at $\mathrm{P}<0.05$.

\section{Results}

\section{EGFR 19del subtypes}

Of the 208 patients, 13 EGFR 19 del subtypes were confirmed in 181 patients (Table 1). Deletions starting from E746 occurred in 143 patients (79.0\%), and starting from L747 occurred in 38 patients (21.0\%). delE746_A750 was found in 130 patients $(71.8 \%)$; deletion starting from E746 with insertions were found in 13 (7.2\%) patients. Deletions starting from $\mathrm{L} 747$ without insertion were found in 16 patients $(8.8 \%)$, and with insertions were found in $22(12.2 \%)$ patients. 
Table 1 EGFR exon 19del subtypes in NSCLC patients

\begin{tabular}{lcc}
\hline No. & Subtypes & $N(\%)$ (total =181) \\
\hline 1 & delE746_A750 & $130(71.8)$ \\
2 & delE746_T751insA & $3(1.7)$ \\
3 & delE746_T751insl & $1(0.6)$ \\
4 & delE746_S752insl & $1(0.6)$ \\
5 & delE746_S752insV & $8(4.4)$ \\
6 & delL747_E749 & $1(0.6)$ \\
7 & delL747_A750insP & $7(3.9)$ \\
8 & delL747_T751insP & $2(1.1)$ \\
9 & delL747_S752 & $1(0.6)$ \\
10 & delL747_P753insQ & $1(0.6)$ \\
11 & delL747_T751 & $14(7.7)$ \\
12 & delL747_P753insS & $11(6.1)$ \\
13 & delL747_A755insSRD & $1(0.6)$ \\
\hline
\end{tabular}

EGFR, epidermal growth factor receptor; 19del, exon 19 deletion; NSCLC, non-small cell lung cancer.

\section{Clinicopathological features of EGFR 19del subtypes}

Since deletions starting from E746 and L747 were two major kind of subtypes, and delE746_A750 was the most frequent subtype, we analyzed the clinicopathological features of the patients according to the two classifications (Table 2). delE746_A750 and deletions starting from E746 were frequently found in female $(\mathrm{P}=0.003$ and $\mathrm{P}=0.013$, respectively) and never smoking patients $(\mathrm{P}=0.002$ and $\mathrm{P}=0.014$, respectively). ARMS was used to detected T790M mutation after patients were resistant to first-line EGFRTKIs. Interestingly, we found that the ratio of T790M was higher in patients harboring delE746_A750 or deletions starting from E746 $(\mathrm{P}=0.001$ and $\mathrm{P}=0.006$, respectively, Table 2). Further, we wanted to explore whether there was sample bias in T790M detecting, and found that there was no significant differences in both classifications (Table 2).

\section{Clinical outcomes of EGFR 19del patients}

For the patients receiving EGFR-TKIs as first-line treatment, the objective response rate (ORR) was $85.4 \%$ (70/82) in delE746_A750 patients and 86.4\% (76/88) in patients with deletions starting from E746 (Table 2). According to the starting codon with or without insertions, we divided EGFR 19del patients into four groups, and found no significant difference between them in PFS (Figure $1 A)$. There was no significant difference between patients with delE746_A750 and other deletions in PFS (12.1 vs. 10.6 months, $\mathrm{P}=0.738$, Figure $1 \mathrm{~B})$, no significant difference between patients with deletions starting from E746 and L747 in PFS (12.1 vs. 10.6 months, $\mathrm{P}=0.816$, Figure 1C), and also no statistical difference between patients with or without insertions (9.5 vs. 12.6 months, $\mathrm{P}=0.102$, Figure 1D). There was no significant difference between deletions starting from E746 with insertion and without insertions (Figure 1E). On the contrary, for patients with deletions starting from L747, those with insertions had statistical shorter PFS then those without (8.3 vs. 15.0 months, $\mathrm{P}=0.017$, Figure $1 F)$. And, patients with deletions starting from L747 with insertions had shorter PFS than all other patients (8.3 vs. 12.6 months, $\mathrm{P}=0.027$, Figure $1 G$ ). Multivariate analysis showed that EGFR 19del subtypes had a marginal effect on PFS ( $\mathrm{P}=0.051$, Table 3). Totally, there were only 24 patients got OS data: 20 with delE746_A750, one with delL747_T751, one with del747_P753insQ, and two with delE746_S752insV. Five of the 20 (25.0\%) patients harboring delE746_A750 had osimertinib treating history, and 2 of the $4(50.0 \%)$ other patients had osimertinib treating history. Patients harboring delE746_A750 had significantly shorter $\mathrm{OS}$ than other patients $(\mathrm{P}=0.022,16.7$ vs. 28.0 months, Figure $1 H$ ).

\section{Impact of different EGFR-TKIs to EGFR 19del patients}

As patients with EGFR 19del could use different EGFRTKIs for first-line treatment, we examined whether there were different clinical outcomes treated with different drugs. The ORR was $83.9 \%$ for gefitinib, $81.8 \%$ for erlotinib, $79.4 \%$ for icotinib and $100 \%$ for afatinib ( $\mathrm{P}=0.784$, Table S2). As there was only one patient treated with afatinib got PFS data, we did not include this patient in the following analysis. For all the other patients, there was no significant difference between different regimens in PFS (11.7 months for gefitinib $v s .8 .5$ months for erlotinib $v s .12 .3$ months for icotinib, $\mathrm{P}=0.123$, Figure $2 A$ ). In patients with del746_ A750, deletions starting from E746 with insertion, deletions starting from $\mathrm{L} 747$ with or without insertion, there were no significant differences between different drugs $(\mathrm{P}=0.061$, $\mathrm{P}=0.156, \mathrm{P}=0.701$, and $\mathrm{P}=0.487$, respectively, Figure $2 B, C, D, E)$. We also investigated whether there was different resistant pattern $(\mathrm{T} 790 \mathrm{M})$ using different drugs, and found no significant difference $(52.4 \%$ in gefitinib, $57.1 \%$ in erlotinib, and $61.5 \%$ in icotinib, $\mathrm{P}=0.837$, Table S3). Further, 
Table 2 Clinicopathological features of EGFR 19del variants

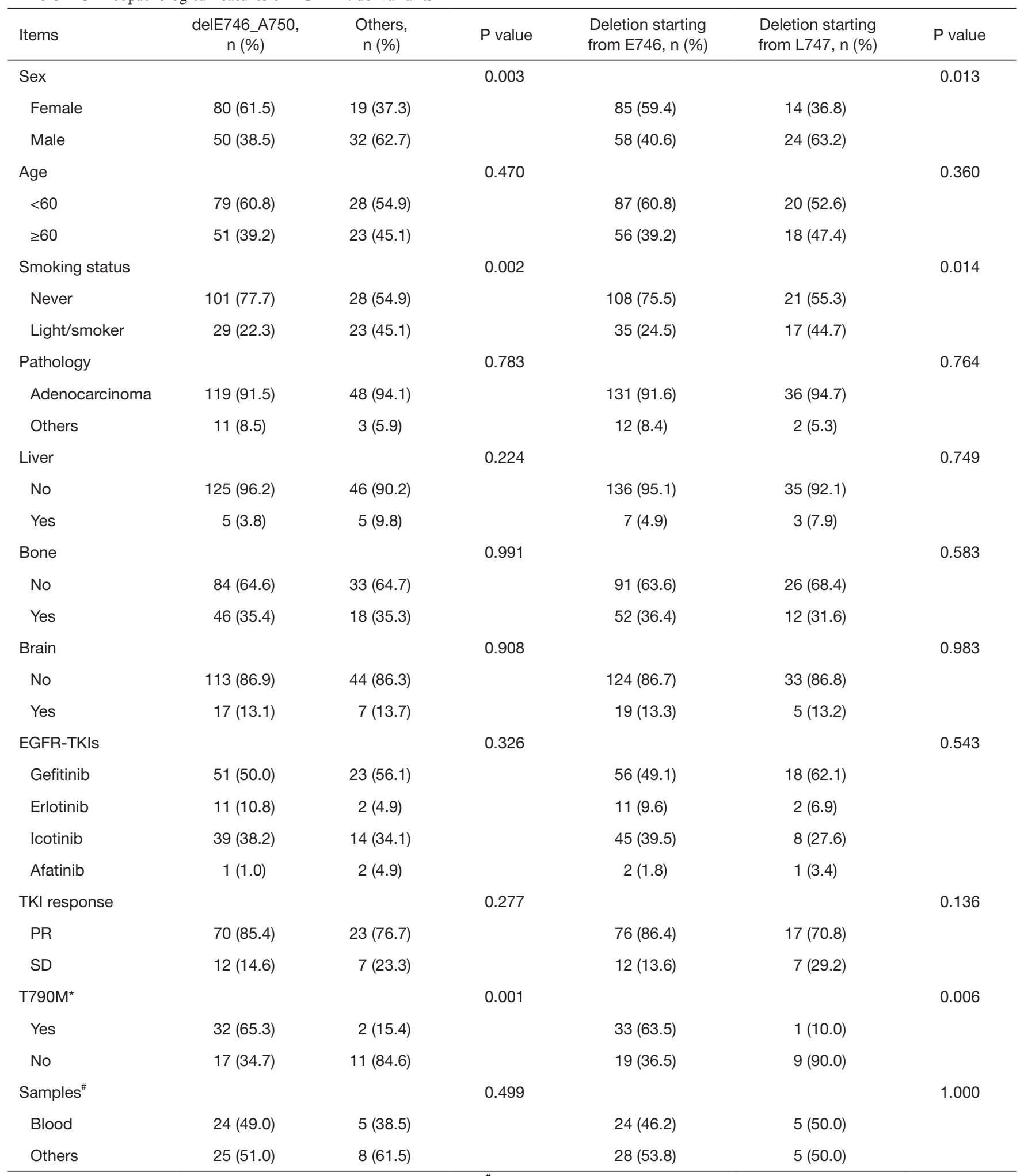

*, Detected after first-line EGFR-TKIs resistance using ARMS; ", samples used for T790M detection. EGFR, epidermal growth factor receptor; 19del, exon 19 deletion; TKIs, tyrosine kinase inhibitors; PR, partial response; SD, stable disease; ARMS, amplification-refractory mutation system. 

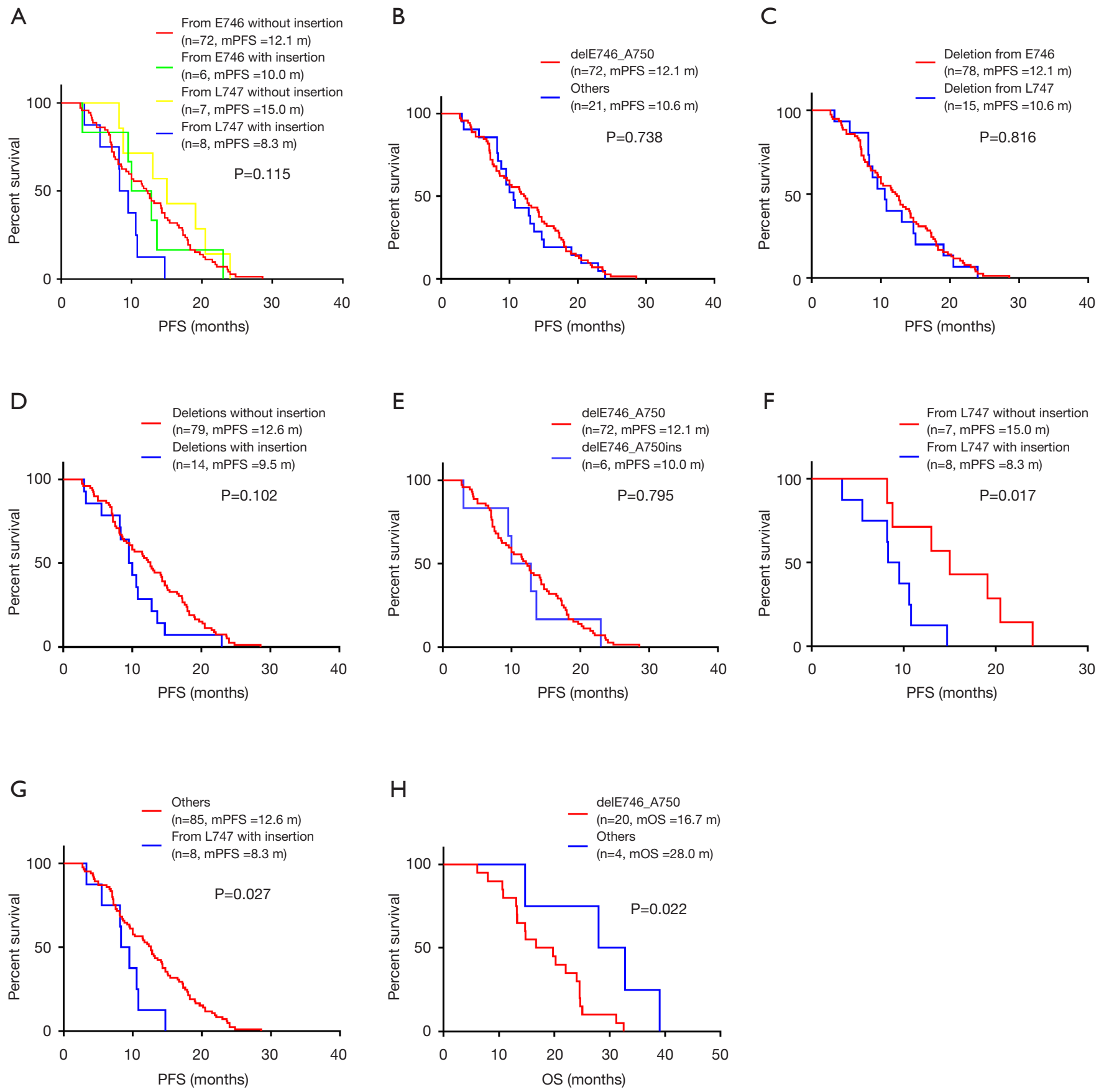

Figure 1 PFS and OS in EGFR 19del patients treated with first-line EGFR-TKIs. (A) PFS of patients harboring deletions starting from E746 or L747 with or without insertions; (B) PFS of patients harboring delE746_A750 or non-delE746_A750; (C) PFS of patients harboring deletions starting from E746 or L747; (D) PFS of patients harboring deletions with or without insertions; (E) PFS of patients harboring delE746_A750 with or without insertion; (F) PFS of patients harboring deletions starting from L747 with or without insertions; (G) PFS of patients harboring deletions starting from L747 with insertions or all other patients except deletions starting from L747 with insertions; (H) OS of patients harboring delE746_A750 or non-delE746_A750. PFS, progression-free survival; OS, overall survival; EGFR, epidermal growth factor receptor; 19del, exon 19 deletion; TKIs, tyrosine kinase inhibitors. 
Table 3 Multivariate analysis of PFS of EGFR 19del patients treated with first-line TKIs

\begin{tabular}{lccc}
\hline Items & $\mathrm{P}$ & $\mathrm{HR}$ & $95 \% \mathrm{Cl}$ \\
\hline Sex & 0.519 & 0.828 & $0.467-1.469$ \\
Age & 0.031 & 1.690 & $1.049-2.722$ \\
Smoking status & 0.748 & 0.901 & $0.478-1.700$ \\
Brain metastasis & 0.340 & 0.762 & $0.436-1.332$ \\
Subtypes & 0.051 & 0.455 & $0.206-1.004$ \\
\hline
\end{tabular}

PFS, progression-free survival; EGFR, epidermal growth factor receptor; 19del, exon 19 deletion; TKls, tyrosine kinase inhibitors.

the first-line PFS was not significantly different in patients harboring T790M or not when resistant to first-line EGFRTKIs (11.5 months for T790M+, 14.7 months for T790M-, $\mathrm{P}=0.092$, Figure $2 F)$.

\section{Osimertinib for EGFR 19del patients}

As we showed the results in Table 2, EGFR T790M mutation distributed differently in EGFR 19 del subtypes. Whether these patients responded differently to osimertinib was analyzed in the study. Among the patients, 25 got PFS data treated with osimertinib: 20 with delE746_A750 deletion, one with delE746_S752insV, two with delL747_T751, and two with delL747_P753insS. There was no significant difference between patients harboring delE746_A750 deletion and other mutations in PFS (4.0 vs. 9.5 months, $\mathrm{P}=0.102$, Figure $3 A$ ), and also no significant difference between patients with deletions starting from E746 and L747 (5.4 vs. 8.0 months, $\mathrm{P}=0.102$, Figure 3B).

\section{Discussion}

Although EGFR mutation patients benefitted a lot from EGFR-TKIs, studies showed that different EGFR mutation patients have different clinical outcomes. Patients harboring 19del could take more advantages than L858R mutation. The EGFR 19 del subtype could also influence patient outcome. In this study, we explored T790M mutation and clinical outcomes to EGFR-TKIs in patients harboring different EGFR 19del subtypes.

Various EGFR 19 del subtypes had been reported $(6,7,12)$. Among these, delE746_A750 was the most frequent subtype, which was in accordance with our results. Deletions starting from E746 and L747 are two frequent subtypes, and the clinical features and outcomes had been compared in former studies $(8,12,13)$. So, in this study, we applied the two classification models on these patients. It was found that delE746_A750 or deletions starting from E746 were most likely to be found in female and never smokers, indicating that even in the EGFR 19del patients there were still different clinical features between them.

The RR and clinical outcomes treated by EGFR-TKIs were compared between different EGFR 19del deletions in former studies, while the results were inconsistent. In these studies, there were mainly three methods to group the 19del patients: deletions from the starting codon E746 or L747 $(8,9,12,13)$; deletions with or without insertion/substitution (9-11); deletions of "LRE" or "non-LRE" $(8,11,12)$. From these studies, we speculated that the clinical outcomes could be different between NSCLC patients harboring different EGFR 19del subtypes, which was that patients with deletions starting from E746 had better outcomes than from L747, and a special subtype of deletion starting from L747 might influence the outcomes. In this study, we found that deletions starting from L747 with insertions had the shortest PFS. However, as the patient number was small, it was not reasonable to further specify which insertion type had the most important influence. Multivariate analysis showed that the deletion subtypes had marginal effect on PFS, indicating that patients with deletions starting from L747 with insertion could be most possibly getting inferior benefit from TKI treatment, and need large samples to confirm the results. Studies explained the relationship between 19del subtypes and outcomes of TKIs treatment in two dimensions: structural features and drug sensitivity. Truini et al. showed the structural features to explain their results (14). However, they mainly analyzed two 19del subtypes. More computational models of mutual interaction between deletion subtypes and EGFR-TKIs will be needed to provide a deep understanding of the interaction. Studies also explored the association from drug sensitivity, they found that delE746_A750 had the lowest $\mathrm{IC}_{50}$ value for target drugs, while others had relative higher $\mathrm{IC}_{50}$ value (22-24). However, the situation was more complicated in patients, as co-alteration of EGFR mutation with other genes could also influence the outcomes (25). We found that patients harboring delE746_A750 had shorter OS than others. As we know, many factors could influence patients' OS, including sample size, follow-up treatments, etc. In this study, we think the most important limitation would be the patient number-a large number of patients and their PFS and OS data are extremely needed. We will follow up the other patients and do further analysis in the future. And it 
A

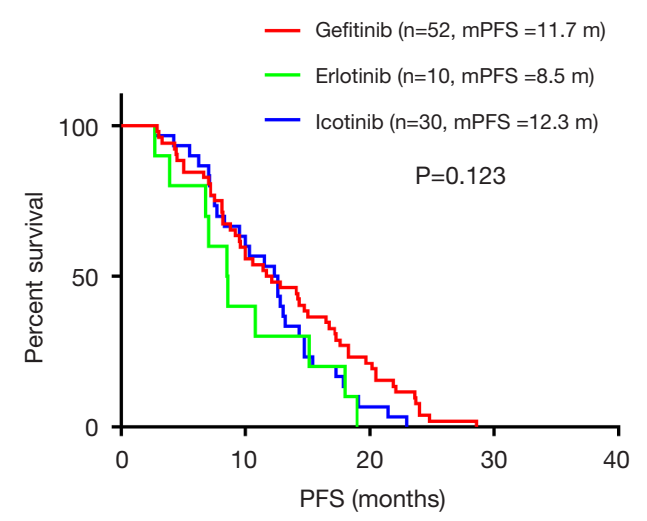

C
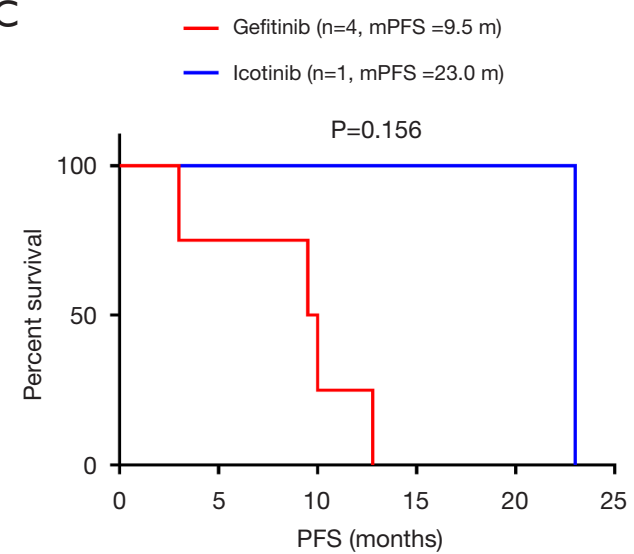

$\mathrm{E}$
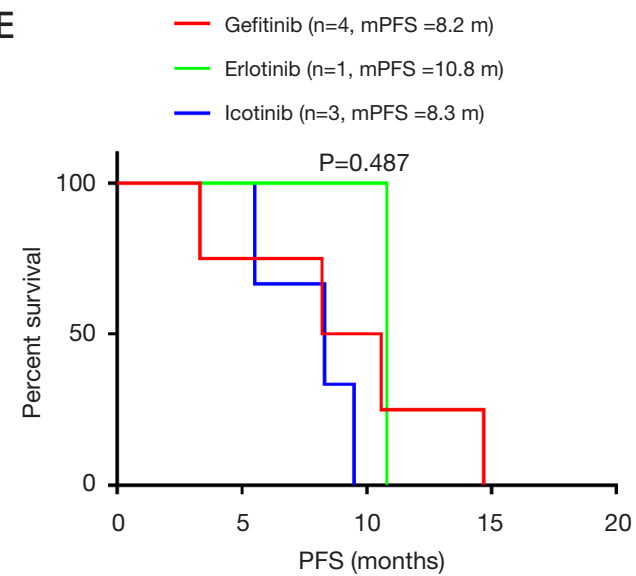

B
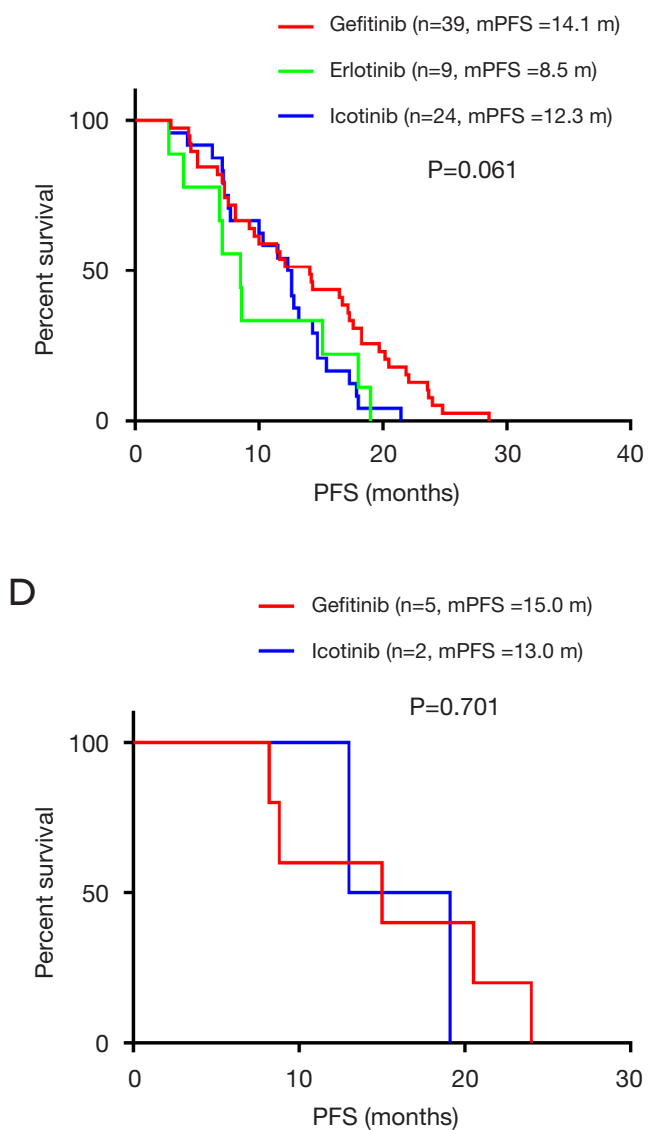

$\mathrm{F}$ - T790M+ $(\mathrm{n}=34, \mathrm{mPFS}=11.5 \mathrm{~m})$ - T790M- $(\mathrm{n}=28, \mathrm{mPFS}=14.7 \mathrm{~m})$

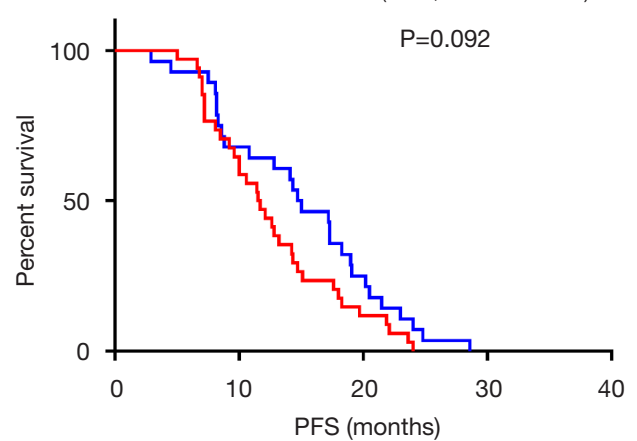

Figure 2 Impact of different EGFR-TKIs to patient PFS. (A) The impact of first-line gefitinib, erlotinib or icotinib to patient PFS; the impact of different drugs to delE746_A750 (B), deletions starting from E746 with insertions (C), deletions starting from L747 without insertions (D), and deletions starting from L747 with insertions (E); (F) PFS of patients harboring T790M (T790M+) or not (T790M-) when treated with first-line EGFR-TKIs. EGFR, epidermal growth factor receptor; TKIs, tyrosine kinase inhibitors; PFS, progression-free survival. 
A

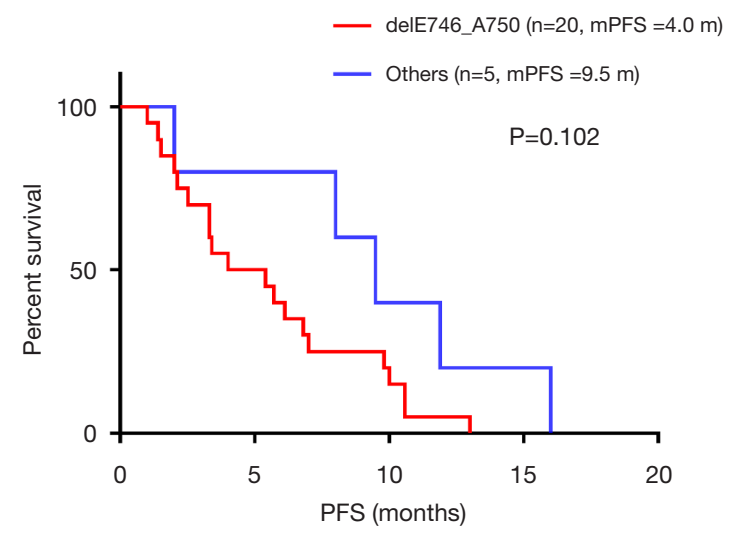

B

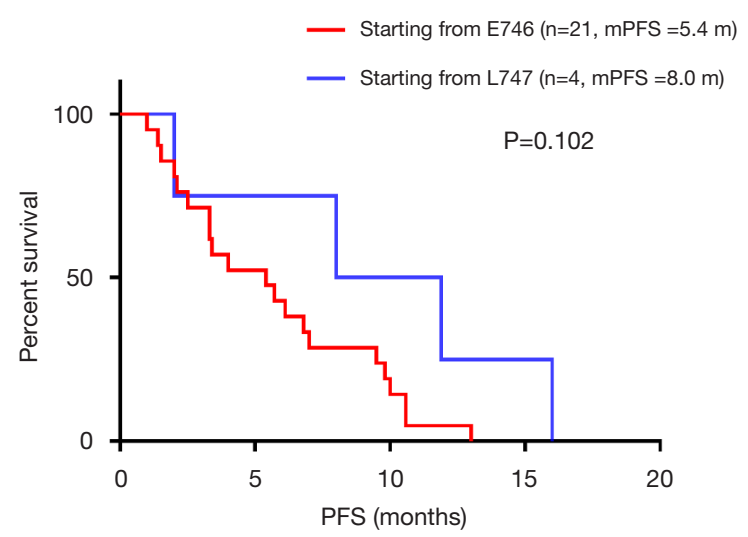

Figure 3 PFS of T790M positive patients treated with osimertinib. (A) PFS of patients harboring delE746_A750 or non-delE746_A750; (B) PFS of patients harboring deletions starting from E746 or L747. PFS, progression-free survival.

also would be better to know comprehensive gene mutation spectrum (e.g., co-alteration or passenger gene mutations) of the patients to understand the impact of $19 \mathrm{del}$ subtypes to patient outcomes.

T790M mutation is the main reason for first/second generation EGFR-TKI resistance. Studies already showed that the incident rate of T790M mutation was higher in EGFR 19del patients than L858R patients $(17,18)$. Huang et al. also reported that T790M mutation had a higher prevalence in delE746_A750 than other 19del subtypes (19), which was in accordance with our results. Besides, we found that it was higher in deletions started from E746 than from L747. We used ARMS PCR to detect T790M for two reasons. Firstly, it is widely and commercially used in China for its convenience and fast speed. Secondly, we only analyzed the data detected by this method to reduce data bias brought by different detecting methods. From former studies (14,22-24), it seemed that delE746_ A750 was more sensitive to EGFR-TKI than deletions starting from E746 with insertions. But for deletions starting from L747, it was complicated. They had similar or higher $\mathrm{IC}_{50}$ than dellE746_A750. It seemed that the more intensive affinity between drugs and EGFR mutants, the less incidence of mutation in the same gene would occur-T790M in this study. Evolution theory, mutation tendency or other theories may be included to clarify the mechanism in the future. Although they had similar outcomes when treated with osimertinib, patients harboring deletions starting from L747 had longer PFS, indicating these patients could more possibly benefit from osimertinib, and also the optimal treatment of osimmertinib or first/second generation TKIs for different 19del subtypes. We will follow up the outcomes of other patients to find if there would be significant difference, and also the outcomes of $19 \mathrm{del}$ patients using osimertinib for first-line therapy.

\section{Conclusions}

In conclusion, different EGFR 19del subtypes of NSCLC patients have different clinical features and outcomes, the mutant rate of T790M after EGFR-TKI resistance could be higher in delE746_A750 or deletions starting from E746 patients, but do not significantly influence outcomes of patients harboring different $19 \mathrm{del}$ subtypes when treated with osimertinib.

\section{Acknowledgments}

Funding: This study was supported by National Natural Science Foundation of China (81871865), Shanghai Key Disciplines Project (2017ZZ02012), and Shanghai Pulmonary Hospital Fund (FK1207 and FK17006).

\section{Footnote}

Reporting Checklist: The authors have completed the STROBE reporting checklist. Available at http://dx.doi. org/10.21037/tlcr-19-359

Data Sharing Statement: Available at http://dx.doi. org/10.21037/tlcr-19-359 
Conflicts of Interest: All authors have completed the ICMJE uniform disclosure form (available at http://dx.doi. org/10.21037/tlcr-19-359). TJ serves as an unpaid editorial board member of Translational Lung Cancer Research from Feb 2018 to Jan 2021. C Zhou serves as an unpaid editorial board member of Translational Lung Cancer Research from Mar 2012 to Mar 2022. The other authors have no conflicts of interest to declare.

Ethical Statement: The authors are accountable for all aspects of the work in ensuring that questions related to the accuracy or integrity of any part of the work are appropriately investigated and resolved. This study was approved by the Ethics Committee of Shanghai Pulmonary Hospital (K19-066Y).

Open Access Statement: This is an Open Access article distributed in accordance with the Creative Commons Attribution-NonCommercial-NoDerivs 4.0 International License (CC BY-NC-ND 4.0), which permits the noncommercial replication and distribution of the article with the strict proviso that no changes or edits are made and the original work is properly cited (including links to both the formal publication through the relevant DOI and the license). See: https://creativecommons.org/licenses/by-nc-nd/4.0/.

\section{References}

1. Mok TS, Wu YL, Thongprasert S, et al. Gefitinib or carboplatin-paclitaxel in pulmonary adenocarcinoma. $\mathrm{N}$ Engl J Med 2009;361:947-57.

2. Zhou C, Wu YL, Chen G, et al. Erlotinib versus chemotherapy as first-line treatment for patients with advanced EGFR mutation-positive non-small-cell lung cancer (OPTIMAL, CTONG-0802): a multicentre, open-label, randomised, phase 3 study. Lancet Oncol 2011;12:735-42.

3. Sakanoue I, Hamakawa H, Kaji R, et al. Sleeve lobectomy for lung adenocarcinoma treated with neoadjuvant afatinib. J Thorac Dis 2018;10:E170-4.

4. Mok TS, Wu YL, Ahn MJ, et al. Osimertinib or platinumpemetrexed in EGFR T790M-positive lung cancer. N Engl J Med 2017;376:629-40.

5. Pisapia P, Rocco D, Pepe F, et al. EGFR exon 19 deletion switch and development of p.L792Q mutation as a new resistance mechanism to osimertinib: a case report and literature review. Transl Cancer Res 2019;8:S64-9.

6. Somatic mutations in epidermal growth factor receptor database (SM-EGFR-DB). Available online: http://www. somaticmutations-egfr.info/index.html

7. COSMIC database. Available online: http://cancer.sanger. ac.uk/cosmic/search?q=egfr

8. Chung KP, Wu SG, Wu JY, et al. Clinical outcomes in non-small cell lung cancers harboring different exon 19 deletions in EGFR. Clin Cancer Res 2012;18:3470-7.

9. Lee VH, Tin VP, Choy TS, et al. Association of exon 19 and 21 EGFR mutation patterns with treatment outcome after first-line tyrosine kinase inhibitor in metastatic nonsmall-cell lung cancer. J Thorac Oncol 2013;8:1148-55.

10. Kaneda T, Hata A, Tomioka H, et al. Possible differential EGFR-TKI efficacy among exon 19 deletional locations in EGFR-mutant non-small cell lung cancer. Lung Cancer 2014;86:213-8.

11. Sutiman N, Tan SW, Tan EH, et al. EGFR mutation subtypes influence survival outcomes following first-line gefitinib therapy in advanced Asian NSCLC patients. J Thorac Oncol 2017;12:529-38.

12. Su J, Zhong W, Zhang X, et al. Molecular characteristics and clinical outcomes of EGFR exon 19 indel subtypes to EGFR TKIs in NSCLC patients. Oncotarget. 2017;8:111246-57.

13. Rossi S, Toschi L, Finocchiaro G, et al. Impact of exon 19 deletion subtypes in EGFR-mutant metastatic non-smallcell lung cancer treated with first-line tyrosine kinase inhibitors. Clin Lung Cancer 2019;20:82-7.

14. Truini A, Starrett JH, Stewart T, et al. The EGFR exon 19 mutant L747-A750>P exhibits distinct sensitivity to tyrosine kinase inhibitors in lung adenocarcinoma. Clin Cancer Res 2019;25:6382-91.

15. Oxnard GR, Arcila ME, Sima CS, et al. Acquired resistance to EGFR tyrosine kinase inhibitors in EGFRmutant lung cancer: distinct natural history of patients with tumors harboring the T790M mutation. Clin Cancer Res 2011;17:1616-22.

16. Lettig L, Sahnane N, Pepe F, et al. EGFR T790M detection rate in lung adenocarcinomas at baseline using droplet digital PCR and validation by ultra-deep next generation sequencing. Transl Lung Cancer Res 2019;8:584-92.

17. Ke EE, Zhou Q, Zhang QY, et al. A higher proportion of the EGFR T790M mutation may contribute to the better survival of patients with exon 19 deletions compared with those with L858R. J Thorac Oncol 2017;12:1368-75.

18. Kaburagi T, Kiyoshima M, Nawa T, et al. Acquired EGFR T790M mutation after relapse following EGFR-TKI therapy: a population-based multi-institutional study. 
Anticancer Res 2018;38:3145-50.

19. Huang YH, Hsu KH, Tseng JS, et al. The association of acquired T790M mutation with clinical characteristics after resistance to first-line epidermal growth factor receptor tyrosine kinase inhibitor in lung adenocarcinoma. Cancer Res Treat 2018;50:1294-303.

20. Li X, Zhao C, Su C, et al. Epidemiological study of HER2 mutations among EGFR wild-type lung adenocarcinoma patients in China. BMC Cancer 2016;16:828.

21. Zhuang X, Zhao C, Li J, et al. Clinical features and therapeutic options in non-small cell lung cancer patients with concomitant mutations of EGFR, ALK, ROS1, KRAS or BRAF. Cancer Med 2019;8:2858-66.

22. Yuza Y, Glatt KA, Jiang J, et al. Allele-dependent variation in the relative cellular potency of distinct EGFR inhibitors.
Cancer Biol Ther 2007;6:661-7.

23. Engelman JA, Zejnullahu K, Gale CM, et al. PF00299804, an irreversible pan-ERBB inhibitor, is effective in lung cancer models with EGFR and ERBB2 mutations that are resistant to gefitinib. Cancer Res 2007;67:11924-32.

24. Furuyama K, Harada T, Iwama E, et al. Sensitivity and kinase activity of epidermal growth factor receptor (EGFR) exon 19 and others to EGFR-tyrosine kinase inhibitors. Cancer Sci 2013;104:584-9.

25. Hong S, Gao F, Fu S, et al. Concomitant genetic alterations with response to treatment and epidermal growth factor receptor tyrosine kinase inhibitors in patients with EGFR-mutant advanced non-small cell lung cancer. JAMA Oncol 2018;4:739-42.
Cite this article as: Zhao C, Jiang T, Li J, Wang Y, Su C, Chen X, Ren S, Li X, Zhou C. The impact of EGFR exon 19 deletion subtypes on clinical outcomes in non-small cell lung cancer. Transl Lung Cancer Res 2020;9(4):1149-1158. doi: 10.21037/ tlcr-19-359 


\section{Supplementary}

Table S1 EGFR 19del subtypes detected by the kit

\begin{tabular}{|c|c|}
\hline No. & Subtypes \\
\hline 1 & delE746_A750 \\
\hline 2 & delL747_P753insS \\
\hline 3 & delE746_T751insl \\
\hline 4 & delE746_T751 \\
\hline 5 & delE746_T751insA \\
\hline 6 & delE746_S752insA \\
\hline 7 & delE746_S752insV \\
\hline 8 & delE746_S752insD \\
\hline 9 & delL747_A750insP \\
\hline 10 & delL747_T751insQ \\
\hline 11 & delL747_E749 \\
\hline 12 & delL747_T751 \\
\hline 13 & delL747_S752 \\
\hline 14 & delL747_A750insP \\
\hline 15 & delL747_P753insQ \\
\hline 16 & delL747_T751insS \\
\hline 17 & delL747_T751 \\
\hline 18 & delL747_T751insP \\
\hline 19 & delL747_T751 \\
\hline 20 & delL747_S752insQ \\
\hline 21 & delL747_A750insP \\
\hline 22 & delL747_K754insQL \\
\hline 23 & delE746_K754insEQHL \\
\hline 24 & delL747_S752insQ \\
\hline 25 & delL747_A755insSRD \\
\hline
\end{tabular}

EGFR, epidermal growth factor receptor; 19del, exon 19 deletion.
Table S2 RR of EGFR 19del patients to different EGFR-TKIs

\begin{tabular}{cccccc}
\hline \multirow{4}{*}{ Items } & \multicolumn{4}{c}{ TKIs } \\
\cline { 2 - 5 } & $\begin{array}{c}\text { Gefitinib, } \\
\mathrm{n}(\%)\end{array}$ & $\begin{array}{c}\text { Erlotinib, } \\
\mathrm{n}(\%)\end{array}$ & $\begin{array}{c}\text { Icotinib, } \\
\mathrm{n}(\%)\end{array}$ & $\begin{array}{c}\text { Afatinib, } \\
\mathrm{n}(\%)\end{array}$ & $\mathrm{P}$ \\
\hline \multicolumn{2}{c}{ TKI response } & & & 0.784 \\
$\mathrm{PR}$ & $52(83.9)$ & $9(81.8)$ & $27(79.4)$ & $2(100.0)$ & \\
$\mathrm{SD}$ & $10(16.1)$ & $2(18.2)$ & $7(20.6)$ & 0 & \\
\hline
\end{tabular}

RR, response rate; EGFR, epidermal growth factor receptor; 19del, exon 19 deletion; TKIs, tyrosine kinase inhibitors; PR, partial response; $\mathrm{SD}$, stable disease.

Table S3 T790M mutation after different EGFR-TKIs treatment

\begin{tabular}{|c|c|c|c|c|}
\hline \multirow[b]{2}{*}{ Items } & \multicolumn{3}{|c|}{ TKIs } & \multirow[b]{2}{*}{$\mathrm{P}$} \\
\hline & $\begin{array}{c}\text { Gefitinib, } \\
\text { n (\%) }\end{array}$ & $\begin{array}{c}\text { Erlotinib, } \\
\text { n (\%) }\end{array}$ & $\begin{array}{c}\text { Icotinib, } \\
\text { n (\%) }\end{array}$ & \\
\hline \multicolumn{4}{|c|}{ T790M mutation } & 0.837 \\
\hline Positive & $22(52.4)$ & $4(57.1)$ & $8(61.5)$ & \\
\hline Negative & $20(47.6)$ & 3 (42.9) & $5(38.5)$ & \\
\hline
\end{tabular}

EGFR, epidermal growth factor receptor; TKIs, tyrosine kinase inhibitors. 